\title{
Correction to: Internet of Things, Smart Spaces, and Next Generation Networks and Systems
}

\author{
Olga Galinina (D), Sergey Andreev (D), Sergey Balandin (D), \\ and Yevgeni Koucheryavy (D)
}

\section{Correction to: \\ O. Galinina et al. (Eds.): \\ Internet of Things, Smart Spaces, and Next Generation \\ Networks and Systems, LNCS 11118, https://doi.org/10.1007/978-3-030-01168-0}

The acknowledgement section of this paper originally stated that "the work was supported by RUDN University Program 5-100." The reference to this was modified to "the publication has been prepared with the support of the RUDN University Program $5-100$ " in the acknowledgement section at the request of the volume editor.

The updated versions of the chapters can be found at https://doi.org/10.1007/978-3-030-01168-0_12 https://doi.org/10.1007/978-3-030-01168-0_32 https://doi.org/10.1007/978-3-030-01168-0_36 https://doi.org/10.1007/978-3-030-01168-0_37 https://doi.org/10.1007/978-3-030-01168-0_40 https://doi.org/10.1007/978-3-030-01168-0_47 https://doi.org/10.1007/978-3-030-01168-0_49 